\title{
Universality in string interactions
}

\author{
Yu-tin Huang, ${ }^{a}$ Oliver Schlotterer ${ }^{b}$ and Congkao Wen ${ }^{c}$ \\ ${ }^{a}$ Department of Physics and Astronomy, National Taiwan University, \\ Taipei 10617, Taiwan, R.O.C. \\ ${ }^{b}$ Max-Planck-Institut für Gravitationsphysik, Albert-Einstein-Institut, \\ 14476 Potsdam, Germany \\ ${ }^{c}$ Dipartimento di Fisica, Università di Roma "Tor Vergata" \\ and I.N.F.N. Sezione di Roma "Tor Vergata", \\ Via della Ricerca Scientifica, 00133 Roma, Italy \\ E-mail: yutinyt@gmail.com, oliver.schlotterer@aei.mpg.de, \\ Congkao.Wen@roma2.infn. it
}

ABSTRACT: In this note, we provide evidence for universality in the low-energy expansion of tree-level string interactions. More precisely, in the $\alpha^{\prime}$-expansion of tree-level scattering amplitudes, we conjecture that the leading transcendental coefficient at each order in $\alpha^{\prime}$ is universal for all perturbative string theories. We have checked this universality up to seven points and trace its origin to the ability to restructure the disk integrals of open bosonic string into those of the superstring. The accompanying kinematic functions have the same low-energy limit and do not introduce any transcendental numbers in their $\alpha^{\prime}$-corrections. Universality in the closed-string sector then follows from KLT-relations.

Keywords: Bosonic Strings, Scattering Amplitudes

ARXIV EPRINT: 1602.01674 


\section{Contents}

1 Introduction 1

2 Open-string amplitudes $\quad 3$

2.1 The bosonic open string 4

2.1.1 Explicit examples 5

2.1.2 Universality and BCJ identities 6

$\begin{array}{lll}3 & \text { Closed-string amplitudes } & 7\end{array}$

$\begin{array}{lll}3.1 & \text { Universality for closed string theories } & 8\end{array}$

$\begin{array}{ll}3.2 \text { Universality of UV completion } & 9\end{array}$

4 Conclusions $\quad 10$

\section{Introduction}

One of the formidable challenges for a theory of quantum gravity is the construction of a gravitational S-matrix which respects unitarity at high energies. Perturbative string theories provide candidate solutions, as its four-point graviton S-matrix is exponentially suppressed in the high-energy limit for fixed-angle scattering [1, 2]. In fact, assuming tree-level causality [3] and unitarity [4] imposes stringent constraints, under which string theories provide the only known analytic solutions so far.

Different string theories are understood to be equivalent through a web of strong-weak dualities which relate different orders in the perturbative expansion [5, 6]. At tree level, however, the low-energy description in the form of an effective action with expansion in curvature tensors and covariant derivatives is largely unconstrained by string dualities. More precisely, the coefficients of these higher-dimensional operators are expected to be distinct for different string theories. Thus, if some of these coefficients turn out to be universal, it is then conceivable that such a phenomenon reflects a deeper principle in the theory of quantum gravity beyond the known dualities.

At low energies, closed-string theories yield an effective action that augments the Einstein-Hilbert term $S_{\mathrm{EH}}$ with higher-dimensional operators. At tree level, type-II superstring theories exhibit the following expansion in the inverse string tension (or cut-off scale) $\alpha^{\prime}$,

$$
\begin{aligned}
S_{\mathrm{eff}}=S_{\mathrm{EH}} & -2 \alpha^{\prime 3} \zeta_{3} e^{-6 \phi} R^{4}-\zeta_{5} \alpha^{15} e^{-10 \phi} D^{4} R^{4} \\
& +\frac{2}{3} \alpha^{\prime 6} \zeta_{3}^{2} e^{-12 \phi} D^{6} R^{4}+\cdots
\end{aligned}
$$

with Einstein-frame conventions for the dilaton couplings $e^{-n \phi}$. The ellipsis $\cdots$ represents loop-corrections and higher-order terms in $\alpha^{\prime}$, while $D^{n} R^{m}$ schematically represent 
contractions of covariant derivatives and Riemann tensors. The tensor structure of each operator as well as its coefficient furnished by multiple zeta values (MZVs)

$$
\zeta_{n_{1}, n_{2}, \ldots, n_{r}} \equiv \sum_{0<k_{1}<k_{2}<\ldots<k_{r}}^{\infty} \frac{1}{k_{1}^{n_{1}} k_{2}^{n_{2}} \ldots k_{r}^{n_{r}}}
$$

can be derived by expanding string-theory graviton amplitudes in $\alpha^{\prime}$. MZVs can be conjecturally categorized according to their transcendental weight $n_{1}+n_{2}+\ldots+n_{r}$ and constitute a fruitful domain of common interest between high-energy physics and number theory. In fact, for type-II theories, the transcendental weight for each coefficient matches the order of $\alpha^{\prime}$. This property will be referred to as uniform transcendentality, and it also exists for open strings in the type-I theory. The type-I effective action is now an expansion in non-abelian field-strength operators $\operatorname{tr}\left(D^{n} F^{m}\right)$. In this light, uniform transcendentality for closed strings is inherited from open strings through the Kawai, Lewellen and Tye (KLT) relations [7].

In this letter, we conjecture that the leading transcendental coefficient at each order in the $\alpha^{\prime}$-expansion of tree-level amplitudes is universal among all perturbative open- and closed-string theories. We have explicitly verified this up to the seven-point level, and the conjectural all-multiplicity extension is further investigated in a companion paper [8]. This also implies that at finite $\alpha^{\prime}$, perturbative closed string amplitudes contain a universal piece that correspond to the UV completion of tree-level Einstein-Hilbert graviton amplitudes. This is given by the type-II theories. For Heterotic and Bosonic closed string theories, this is augmented by separate terms that correspond to UV completions of amplitudes generated by higher dimensional operators such as $R^{2}, R^{2} \phi$ and $R^{3}$, where $\phi$ can be the dilaton or the Tachyon. This remarkable property can be best understood by inspecting the world-sheet correlator of the open-string amplitudes.

It was shown in [9] that the $n$-point tree amplitude of the open superstring can be cast into an $(n-3)$ ! basis of disk integrals, each augmented with Yang-Mills tree amplitudes of different color-orderings. These basis integrals exhibit uniform transcendentality upon $\alpha^{\prime}$-expansion, see e.g. [10] for a proof. We claim that bosonic open-string disk integral can be cast into the very same basis where - in contrast to the superstring - the augmented function depends on $\alpha^{\prime}$. More precisely, this augmented function contains apart from the Yang-Mills tree amplitude, additional rational functions that contain Tachyon poles. Thus in their low-energy limit $\alpha^{\prime} \rightarrow 0$, the $\alpha^{\prime}$-corrections of the kinematic functions exclusively involve rational numbers upon Taylor-expansion, i.e. they do not carry any transcendental weight. This implies that the resulting $\alpha^{\prime}$-expansion of the bosonic string amplitude will have the same leading transcendental pieces as found for the superstring.

The same property can be extended to closed strings by utilizing the KLT-relations [7], which assemble closed-string tree amplitudes from products of two open-string trees. The accompanying sin-functions with $\alpha^{\prime}$-dependent arguments do not alter the uniform transcendentality of the type-II theory. Different double-copies of open bosonic strings and superstrings give rise to three different closed-string theories - bosonic, heterotic and type-II superstrings. Their tree amplitudes are governed by a universal basis of $(n-3) ! \times(n-3)$ ! 
integrals of uniform transcendentality inherited from the open-string constituents. Only the kinematic coefficients differ between the theories, where the additional $\alpha^{\prime}$-corrections specific to open bosonic strings do not introduce any transcendental weight and thereby do not affect the leading-transcendental piece. This completes the argument for universality in closed-string interactions, namely for the $\mathcal{O}\left(\alpha^{\prime n}\right)$ order of the effective action, the weight- $n$ coefficient is universal for all perturbative closed-string theories. More over, this representation also allows us to separate a piece of the closed string graviton amplitude that is universal and is given by the type II theories.

This paper is organized as follows: in the next section, we give a brief review of the organization of open superstring amplitude as a matrix of disk integrals multiplied by the basis of Yang-Mills amplitude. We will show that through a series of Integration By Parts (IBP) identity, the bosonic string amplitude can be cast into the very same same form with identical integral matrix, with $\alpha^{\prime}$ dependent basis. In section 3 , we discussed the implication of this property for the $\alpha^{\prime}$ expansion of closed strings, utilising the KLTrelations. Finally, we show that the closed string graviton amplitude for all perturbative string theories can be naturally separated into a piece that can be identified as the UV completion of pure tree-level Einstein-Hilbert graviton amplitudes, which is given by that of type II theories.

\section{Open-string amplitudes}

The tree-level amplitude for $n$ gluon-multiplet states in open superstring theory can be conveniently written as [9]

$$
\mathcal{A}^{S}\left(1,2_{\rho}, \ldots,(n-2)_{\rho}, n-1, n ; \alpha^{\prime}\right)=\sum_{\sigma \in S_{n-3}} F_{\rho}{ }^{\sigma}\left(\alpha^{\prime}\right) A_{\mathrm{YM}}\left(1,2_{\sigma}, \ldots,(n-2)_{\sigma}, n-1, n\right),
$$

where $\mathcal{A}^{S}$ and $A_{\mathrm{YM}}$ indicate color-ordered amplitudes of the superstring and super YangMills field theory, respectively. The ordering of the two amplitudes does not have to be identical. More precisely the two ordering are labeled by $\rho, \sigma$ respectively, which denote the $(n-3)$ ! distinct permutations with legs $1, n-1, n$ held fixed. We will use $j_{\rho} \equiv \rho(j)$ to represent the $j$-th element in the $\rho$ ordering. The functions $F_{\rho}{ }^{\sigma}\left(\alpha^{\prime}\right)$ are disk integrals that capture the $\alpha^{\prime}$-dependence,

$$
\begin{aligned}
& F_{\rho}{ }^{\sigma}\left(\alpha^{\prime}\right) \equiv \int \mathrm{d} z_{2} \ldots \mathrm{d} z_{n-2} \prod_{i<l}^{n}\left|z_{i l}\right|^{s_{i l}} \sigma \\
& 0 \leq z_{2_{\rho}} \leq z_{3_{\rho}} \leq \ldots \leq z_{(n-2) \rho} \leq 1
\end{aligned}\left\{\prod_{k=2}^{n-2} \sum_{m=1}^{k-1} \frac{s_{m k}}{z_{k m}}\right\},
$$

with $z_{i j} \equiv z_{i}-z_{j}$. We see that $\rho$ denotes the ordering of the integration region. We fix the $\mathrm{SL}(2)$ symmetry of the disk by setting $\left(z_{1}, z_{n-1}, z_{n}\right)=(0,1, \infty)$, and we use dimensionless Mandelstam invariants

$$
s_{i j \ldots l} \equiv \alpha^{\prime}\left(k_{i}+k_{j}+\ldots+k_{l}\right)^{2} .
$$

When viewed as an $(n-3) ! \times(n-3) !$ matrix, the row- and column indices $\rho$ and $\sigma$ of $F_{\rho}{ }^{\sigma}$ label different integration domains and integrands, respectively, where $\sigma$ acts on the subscripts 
within the curly bracket in (2.2). Note that the field-theory limit is recovered as $F_{\rho}{ }^{\sigma}\left(\alpha^{\prime}\right)=$ $\delta_{\rho}{ }^{\sigma}+\mathcal{O}\left(\alpha^{\prime 2}\right)$, and the $(n-3)$ !-vector in (2.1) furnishes a basis of string subamplitudes under monodromy relations $[11,12]$.

The $\alpha^{\prime}$-expansion of the integrals in (2.2) yields MZVs (1.2) whose transcendental weight matches the degree of the accompanying polynomials in $s_{i j}$. Since $A_{\mathrm{YM}}$ do not depend on $\alpha^{\prime}$, uniform transcendentality of the integrals propagates to the open-superstring amplitude (2.1). Initially addressed via hypergeometric functions [13, 14], the $\alpha^{\prime}$-corrections of $F_{\rho}{ }^{\sigma}\left(\alpha^{\prime}\right)$ at any multiplicity can be recursively generated from the Drinfeld associator [10].

Once undoing the above choice of $\mathrm{SL}(2)$ frame, the functions (2.2) can be identified as a superposition of $(n-3)$ ! "single-cycle" disk integrals,

$$
Z_{\rho}\left(1_{\sigma}, 2_{\sigma}, \ldots, n_{\sigma}\right) \equiv \int \frac{\mathrm{d} \mu_{n}(\rho)}{\sigma\left(z_{12} z_{23} \ldots z_{n 1}\right)},
$$

where $\sigma$ and $\rho$ now act on all external legs in the integrand and the integration domain, respectively, and the measure is given by

$$
\int \mathrm{d} \mu_{n}(\rho) \equiv \int_{-\infty<z_{1} \leq z_{2} \leq \ldots \leq z_{n_{\rho}}<\infty} \frac{\mathrm{d} z_{1} \mathrm{~d} z_{2} \ldots \mathrm{d} z_{n}}{\operatorname{vol}(\operatorname{SL}(2))} \prod_{i<l}^{n}\left|z_{i l}\right|^{s_{i l}} .
$$

The integral reductions performed in [9] rely on partial-fraction manipulations and integrations by parts (IBP) among $Z_{\rho}\left(1_{\sigma}, \ldots, n_{\sigma}\right)$. At fixed $\rho$, these integral relations for different choices of $\sigma$ can be identified with the KK- and BCJ-relations [15-17] of $A_{\mathrm{YM}}(\ldots)$ [18]. However, as already exploited in a superstring context $[9,19]$, IBP additionally allows to address closed subcycles of $z_{i j}$ in the integrand such as double poles $z_{i j}^{-2}$. Extending these techniques to gluon amplitudes of the bosonic string yields our main result to be reported in the following.

\subsection{The bosonic open string}

The tree-amplitude prescription for $n$-gluon scattering in the bosonic string introduces significantly more rational functions of $z_{i j}$ of suitable SL(2) weight than captured by the single cycles in (2.4). In particular, one obtains more terms involving higher order poles as well as multicycle denominators. Still, repeated use of IBP is expected to reduce all of them to the single-cycle form and thereby to the same integral basis as seen in (2.1) and (2.2). For example, at four-points, all double poles can be reduced by using the following identity:

$$
\int \frac{\mathrm{d} \mu_{4}(\rho)}{z_{14}^{2} z_{23}^{2}}=\frac{s_{12} Z_{\rho}(1,2,3,4)}{1-s_{23}} .
$$

The denominator on the right-hand side signals tachyon exchange specific to the bosonic string and can be expanded as a geometric series $\left(1-s_{i j}\right)^{-1}=\sum_{k=0}^{\infty} s_{i j}^{k}$. Note that for open superstring, while such double cycle denominators are also present, the OPE among supersymmetric vertex operators guarantees that tachyon poles as in (2.6) are suppressed by numerators $1-s_{i j}$. For explicit examples see e.g. [9, 19]. 
Extending the integral reduction along the lines of (2.6) to arbitrary multiplicity leads us to conjecture the following structure for the $n$-gluon tree in bosonic string theory:

$$
\mathcal{A}^{B}\left(1,2_{\rho}, \ldots,(n-2)_{\rho}, n-1, n ; \alpha^{\prime}\right)=\sum_{\sigma \in S_{n-3}} F_{\rho}{ }^{\sigma}\left(\alpha^{\prime}\right) B\left(1,2_{\sigma}, \ldots,(n-2)_{\sigma}, n-1, n ; \alpha^{\prime}\right) .
$$

In comparison to the superstring result (2.1), the kinematic factors $A_{\mathrm{YM}}(\ldots)$ are replaced by more general and $\alpha^{\prime}$-dependent objects $B\left(\ldots ; \alpha^{\prime}\right)$. Both of them are rational functions of $s_{i j}$ and multilinear in the polarizations $e_{j}$ entering via $\left(e_{i} \cdot e_{j}\right)$ and $\left(e_{i} \cdot k_{j}\right)$, and crucially do not carry any transcendental weights. Upon $\alpha^{\prime}$-expansion, the leading term reproduces Yang-Mills tree amplitudes, and is therefore identical to that of the superstring, i.e.

$$
B\left(1, \ldots, n ; \alpha^{\prime}\right)=A_{\mathrm{YM}}(1, \ldots, n)+\sum_{k=1}^{\infty}\left(2 \alpha^{\prime}\right)^{k} B_{k}(1, \ldots, n) .
$$

At generic multiplicity $n$, the $B_{k}(\ldots)$ 's have homogeneity degree $4-n+2 k$ in momenta.

The simplest instances of the subleading terms occur at the three-point level and signal the $F^{3}$ interaction specific to the bosonic string,

$$
B_{1}(1,2,3)=\left(e_{1} \cdot k_{2}\right)\left(e_{2} \cdot k_{3}\right)\left(e_{3} \cdot k_{1}\right), \quad B_{k \geq 2}(1,2,3)=0 .
$$

The higher-point case requires integral reductions as in (2.6), and the resulting geometric series yield non-zero $B_{k}(\ldots)$ for any value of $k$.

\subsubsection{Explicit examples}

A. Four-points. In the case of $n=4$, we find

$$
\begin{aligned}
B\left(1,2,3,4 ; \alpha^{\prime}\right)= & A_{\mathrm{YM}}(1,2,3,4)+\left(2 \alpha^{\prime}\right)^{2} \\
& \times s_{13}\left[\left(\frac{f_{12} f_{34}}{s_{12}^{2}\left(1-s_{12}\right)}+\operatorname{cyc}(2,3,4)\right)-\frac{g_{1} g_{2} g_{3} g_{4}}{s_{12}^{2} s_{13}^{2} s_{14}^{2}}\right],
\end{aligned}
$$

with gauge invariant constituents $f_{i j} \equiv\left(e_{i} \cdot e_{j}\right)\left(k_{i} \cdot k_{j}\right)-\left(k_{i} \cdot e_{j}\right)\left(k_{j} \cdot e_{i}\right)$ and $g_{i} \equiv\left(k_{i-1}\right.$. $\left.e_{i}\right) s_{i, i+1}-\left(k_{i+1} \cdot e_{i}\right) s_{i-1, i}$. Note that both $s_{i j}$ and $g_{i}$ carry a power of $\alpha^{\prime}$ when extracting the $B_{k}(1,2,3,4)$ 's from the second line of $(2.10)$.

B. Five-points. At five points, after partial fractional manipulations, we require following two identities to reduce all the integrals to a single-cycle basis (2.4):

$$
\begin{aligned}
\int \frac{\mathrm{d} \mu_{5}(\rho)}{z_{23}^{2}\left(z_{15} z_{54} z_{41}\right)}= & \frac{s_{12} Z_{\rho}(1,2,3,5,4)-(1 \leftrightarrow 4)}{s_{23}-1} \\
\int \frac{\mathrm{d} \mu_{5}(\rho) z_{25}}{z_{23}^{2} z_{15}^{2} z_{24} z_{45}}= & \frac{s_{13} Z(1,3,2,4,5)}{1-s_{51}} \\
& +\frac{s_{14}}{1-s_{51}}\left[\frac{s_{12} Z_{\rho}(1,2,3,5,4)-(1 \leftrightarrow 4)}{s_{23}-1}\right] .
\end{aligned}
$$

The resulting form for $B\left(1,2,3,4,5 ; \alpha^{\prime}\right)$ is rather lengthy, and an auxiliary mathematica notebook containing the full expression is attached. 
C. Six-points. To arrive at (2.7) at six points, we find that after partial fractions, besides the single-cycle basis we encounter integrals of following forms,

$$
\begin{aligned}
& \frac{1}{\left(z_{23} z_{34} z_{42}\right)\left(z_{15} z_{56} z_{61}\right)}, \quad \frac{1}{z_{23}^{2}\left(z_{14} z_{46} z_{65} z_{51}\right)}, \\
& \frac{1}{z_{12}^{2} z_{34}^{2} z_{56}^{2}}, \quad \frac{z_{36}}{z_{23}^{2} z_{56}^{2} z_{13} z_{14} z_{46}}, \quad \frac{z_{26}}{z_{23} z_{34} z_{42} z_{56}^{2} z_{12} z_{16}} \text {. }
\end{aligned}
$$

We have checked that indeed all above integrals can be reduced to single-cycle integral via IBP, for instance,

$$
\begin{aligned}
\int \frac{\left(s_{234}-1\right) \mathrm{d} \mu_{6}(\rho)}{\left(z_{23} z_{34} z_{42}\right)\left(z_{15} z_{56} z_{61}\right)}= & s_{13} Z_{\rho}(1,3,4,2,6,5) \\
& -s_{35} Z_{\rho}(1,6,2,4,3,5)-(3 \leftrightarrow 4) .
\end{aligned}
$$

Note that all identities of (2.6), (2.11) and (2.13) can alternatively be derived by imposing linearized gauge invariance under $e_{j} \rightarrow k_{j}$, and the same is believed to hold for the integral reduction at arbitrary multiplicity.

The analogous seven-point checks to arrive at (2.7) have been performed as well.

\subsubsection{Universality and BCJ identities}

It is crucial to note that no MZVs or transcendental weight accompany the $\alpha^{\prime}$-dependence from $B\left(\ldots ; \alpha^{\prime}\right)$, due to the fact that it is a rational function. Given the uniform transcendentality of the $F_{\rho}{ }^{\sigma}\left(\alpha^{\prime}\right)$ and the absence of negative powers of $\alpha^{\prime}$ in the kinematic factor (2.8), the transcendental weight cannot exceed the accompanying order in $\alpha^{\prime}$ within the bosonic-string amplitude. At fixed order in $\alpha^{\prime}$, the leading-transcendental part of the open bosonic string follows from picking up $B\left(\ldots ; \alpha^{\prime}\right) \rightarrow A_{\mathrm{YM}}(\ldots)$ in $(2.8)$ and therefore must agrees with the superstring amplitude. This leads to the conclusion that the leadingtranscendental pieces of the tree-level $\alpha^{\prime}$-expansion and the resulting $\operatorname{tr}\left(D^{n} F^{m}\right)$ interactions are universal in open-string theories.

Although the kinematic factors $B_{k}(\ldots)$ in (2.8) differ from $A_{\mathrm{YM}}(\ldots)$ in tensor structure and mass dimension, we will now argue that they obey the same KK- and BCJ-relations [1517]. The universal monodromy relations [11, 12] among bosonic-string subamplitudes have to hold separately at each order in $\alpha^{\prime}$ and along with each transcendentality. Hence, inserting (2.7) into the lowest-transcendentality pieces of the monodromy relations and identifying $B_{0}(\ldots) \equiv A_{\mathrm{YM}}(\ldots)$ yields

$$
\begin{aligned}
0= & B_{k}(1,2, \ldots, n)+B_{k}(2,1,3, \ldots, n)+B_{k}(2,3,1, \ldots, n) \\
& +\ldots+B_{k}(2,3, \ldots, n-1,1, n) \\
0= & s_{12} B_{k}(2,1,3, \ldots, n)+\left(s_{12}+s_{13}\right) B_{k}(2,3,1,4, \ldots, n) \\
& +\ldots+\left(s_{12}+s_{13}+\ldots+s_{1, n-1}\right) B_{k}(2,3, \ldots, n-1,1, n)
\end{aligned}
$$

for any $k \geq 0$. The idea of imposing monodromy relations order by order has been exploited in [20] to derive BCJ-relations for subamplitudes of the $F^{3}$ operators as well as the supersymmetrized $D^{2} F^{4}+F^{5}$. Moreover, an all-order argument for single-trace gluon 
amplitudes of the heterotic string has been given in [21]. By the same reasoning, (2.14) can be extended to an infinity of $\alpha^{\prime}$-corrections labelled by $j_{i} \in 2 \mathbb{N}+1$ :

$$
\begin{aligned}
B_{k}^{j_{1} j_{2} \ldots j_{p}} & \left(1,2_{\sigma}, \ldots,(n-2)_{\sigma}, n-1, n\right) \\
& \equiv \sum_{\tau \in S_{n-3}}\left(M_{j_{1}} M_{j_{2}} \ldots M_{j_{p}}\right)_{\sigma}{ }^{\tau} B_{k}\left(1,2_{\tau}, \ldots,(n-2)_{\tau}, n-1, n\right) .
\end{aligned}
$$

The $(n-3) ! \times(n-3)$ ! matrix $M_{j}$ is the coefficient of $\zeta_{j}$ when casting the $\alpha^{\prime}$-expansion of $F_{\rho}{ }^{\sigma}$ in $(2.2)$ into a conjectural basis of MZVs w.r.t. rational numbers $\mathbb{Q}[22]$. The entries of $M_{j}$ are degree- $j$ polynomials in $s_{p q}$, see [23] for examples at multiplicity $n \leq 7$.

Note that the symmetry properties $(2.14)$ of $B_{k}(\ldots)$ and their deformations $B_{k}^{j_{1} \ldots j_{p}}(\ldots)$ in (2.15) are inevitable to verify permutation invariance of the world-sheet integrand for the bosonic-string amplitude along with each transcendentality and order in $\alpha^{\prime}$.

\section{Closed-string amplitudes}

Closed-string amplitudes at tree level can be obtained from squares of open-string amplitudes through the KLT-relations [7]. The accompanying factors of $\sin \left(\pi s_{i j}\right)$ conspire with the $\alpha^{\prime}$-expansion of the open string such as various MZVs including all $\zeta_{2 n}$ cancel in a suitable basis w.r.t. $\mathbb{Q}[22,24]$. The selection rules were identified in $[25]$ with the single-valued projection of MZVs [26, 27]. A representation of the massless closed-superstring tree $\mathcal{M}_{n}^{S}$ which manifests the effect of these cancellations has been firstly given in [22]:

$$
\begin{aligned}
\mathcal{M}_{n}^{S}\left(\alpha^{\prime}\right)= & \sum_{\sigma, \rho, \tau \in S_{n-3}} \tilde{A}_{\mathrm{YM}}\left(1,2_{\sigma}, \ldots,(n-2)_{\sigma}, n, n-1\right)\left(S_{0}\right)_{\sigma}{ }^{\rho} \\
& \times G_{\rho}{ }^{\tau}\left(\alpha^{\prime}\right) A_{\mathrm{YM}}\left(1,2_{\tau}, \ldots,(n-2)_{\tau}, n-1, n\right) .
\end{aligned}
$$

The polarizations of the type-II supergravity multiplets stem from tensor products of the gauge-multiplet polarizations in $\tilde{A}_{\mathrm{YM}}$ and $A_{\mathrm{YM}}$. The matrix $S_{0}$ has entries of order $\left(k_{i} \cdot k_{j}\right)^{n-3}$ and appears in the momentum-kernel representation [28] of the KLT-formula for supergravity trees [29].

In order to clarify the relation between $F\left(\alpha^{\prime}\right)$ and $G\left(\alpha^{\prime}\right)$, we recall the organization of the open-string $\alpha^{\prime}$-expansion [22]

$$
\begin{aligned}
F\left(\alpha^{\prime}\right)= & 1+\zeta_{2} P_{2}+\zeta_{3} M_{3}+\zeta_{2}^{2} P_{4}+\zeta_{5} M_{5}+\zeta_{2} \zeta_{3} P_{2} M_{3}+\zeta_{2}^{3} P_{6}+\frac{1}{2} \zeta_{3}^{2} M_{3} M_{3}+\zeta_{7} M_{7} \\
& +\zeta_{2} \zeta_{5} P_{2} M_{5}+\zeta_{2}^{2} \zeta_{3} P_{4} M_{3}+\zeta_{2}^{4} P_{8}+\zeta_{3} \zeta_{5} M_{5} M_{3}+\frac{1}{2} \zeta_{2} \zeta_{3}^{2} P_{2} M_{3} M_{3}+\frac{1}{5} \zeta_{3,5}\left[M_{5}, M_{3}\right] \\
& +\ldots+\left(9 \zeta_{2} \zeta_{9}+\frac{6}{25} \zeta_{2}^{2} \zeta_{7}-\frac{4}{35} \zeta_{2}^{3} \zeta_{5}+\frac{1}{5} \zeta_{3,3,5}\right)\left[M_{3},\left[M_{5}, M_{3}\right]\right]+\ldots
\end{aligned}
$$

where the entries of the $(n-3) ! \times(n-3)$ ! matrices $P_{w}, M_{w}$ are degree $w$ polynomials in $s_{i j}$ with rational coefficients. Once the MZVs are expressed in terms of their conjectural basis over $\mathbb{Q}$, only one independent matrix $P_{w}$ or $M_{w}$ occurs at each weight $w$ along with the primitive $\zeta_{w}$. Any other combination of MZVs is accompanied by a matrix product as seen in (3.2) recycling information from lower weights. The rational prefactors $\frac{1}{5}, \frac{6}{25}, \ldots$ 
along with the matrix commutators $\left[M_{5}, M_{3}\right], \ldots$ can be understood from the coproduct of MZVs which can only be rigorously defined for their motivic versions. A description of motivic MZVs which lead to a rewriting of (3.2) with unit rational coefficients can be found in $[22]$.

The $\alpha^{\prime}$-expansion of the closed string in terms of $G$ in (3.1) only involves a subset of the terms in (3.2) [22],

$$
\begin{aligned}
G\left(\alpha^{\prime}\right)= & 1+2 \zeta_{3} M_{3}+2 \zeta_{5} M_{5}+2 \zeta_{3}^{2} M_{3} M_{3}+2 \zeta_{7} M_{7}+2 \zeta_{3} \zeta_{5}\left\{M_{3}, M_{5}\right\} \\
& +\ldots+2\left(9 \zeta_{2} \zeta_{9}+\frac{6}{25} \zeta_{2}^{2} \zeta_{7}-\frac{4}{35} \zeta_{2}^{3} \zeta_{5}+\frac{1}{5} \zeta_{3,3,5}\right)\left[M_{3},\left[M_{5}, M_{3}\right]\right]+\ldots
\end{aligned}
$$

The matrices $P_{w}$ associated with even weight and powers $\zeta_{2}^{w / 2}$ cancel from non-trivial conspirations in the KLT-formula. Moreover, the properties of the $M_{w}$ matrices additionally eliminate $\zeta_{3,5}$ and many further higher-depth MZVs from the open string expansion (3.2). These selection rules have been identified in [25] with the single-valued projection of MZVs,

$$
G\left(\alpha^{\prime}\right)=\operatorname{sv}\left(F\left(\alpha^{\prime}\right)\right) \text {, }
$$

see $[26,27]$ for further mathematical background.

Together with the polarization-dependence from $\tilde{A}_{\mathrm{YM}}, A_{\mathrm{YM}}$, they encode the tensor contractions of the $D^{n} R^{m}$ operators in the tree-level effective action to the orders seen in (1.1). Given the ubiquitous matrix products with summations over permutations in $S_{n-3}$, we will drop indices henceforth and rewrite (3.1) in the condensed notation

$$
\mathcal{M}_{n}^{S}\left(\alpha^{\prime}\right)=\tilde{A}_{\mathrm{YM}} \cdot S_{0} \cdot G\left(\alpha^{\prime}\right) \cdot A_{\mathrm{YM}},
$$

where the vectors $\tilde{A}_{\mathrm{YM}}$ and $A_{\mathrm{YM}}$ are understood to be in the different $(n-3)$ !-bases spelt out in (3.1).

\subsection{Universality for closed string theories}

As exploited in [21] for the heterotic string, the above structure and $\alpha^{\prime}$-expansion of typeII closed-string amplitudes are a property of the sphere integrals involving two copies of the integrands in (2.2). Accordingly, the results on the integrals can be exploited in further contexts such as gravitational tree amplitudes $\mathcal{M}_{n}^{H}$ or $\mathcal{M}_{n}^{B}$ in the heterotic or the closed bosonic string which rest on one or two copies of the bosonic-string integrand in (2.7). The only modification as compared to the superstring (3.1) is an exchange of $A_{\mathrm{YM}}(\ldots) \leftrightarrow B\left(\ldots, \alpha^{\prime}\right)$,

$$
\begin{aligned}
& \mathcal{M}_{n}^{H}\left(\alpha^{\prime}\right)=\tilde{A}_{\mathrm{YM}} \cdot S_{0} \cdot G\left(\alpha^{\prime}\right) \cdot B\left(\alpha^{\prime}\right) \\
& \mathcal{M}_{n}^{B}\left(\alpha^{\prime}\right)=\tilde{B}\left(\alpha^{\prime}\right) \cdot S_{0} \cdot G\left(\alpha^{\prime}\right) \cdot B\left(\alpha^{\prime}\right),
\end{aligned}
$$

where the same bases of color-orderings spelt out in (3.1) are used for $\tilde{B}\left(\alpha^{\prime}\right), \tilde{A}_{\mathrm{YM}}$ and $B\left(\alpha^{\prime}\right), A_{\mathrm{YM}}$, respectively. The single-valued projection [26, 27] allows for alternative representations in terms of $G\left(\alpha^{\prime}\right) \cdot B\left(\alpha^{\prime}\right)=\operatorname{sv}\left(\mathcal{A}^{B}\left(\alpha^{\prime}\right)\right)$, generalizing the analogous type-II result of [25] based on $G\left(\alpha^{\prime}\right) \cdot A_{\mathrm{YM}}=\operatorname{sv}\left(\mathcal{A}^{S}\left(\alpha^{\prime}\right)\right)$. 
Clearly, the Einstein-Hilbert interaction can be recovered from (3.6) and (3.7) at leading order in $\alpha^{\prime}$ where $G\left(\alpha^{\prime}\right) \rightarrow 1, B\left(\alpha^{\prime}\right) \rightarrow A_{\mathrm{YM}}$ and $\tilde{B}\left(\alpha^{\prime}\right) \rightarrow \tilde{A}_{\mathrm{YM}}$. The $R^{2}$-correction at subleading order is recovered from instead setting $B\left(\alpha^{\prime}\right) \rightarrow 2 \alpha^{\prime} B_{1}$. Note that, by levelmatching, the tachyonic poles of the form $\left(1-s_{i j \ldots l}\right)^{-1}$ in $B\left(\alpha^{\prime}\right)$ of the heterotic amplitude (3.6) are cancelled by corresponding zeros in the entries of $G\left(\alpha^{\prime}\right)$ as $s_{i j \ldots l} \rightarrow 1$. The structure of (3.6) is expected to capture multitrace interactions in the gauge sector of the heterotic string under appropriate replacement of $B\left(\alpha^{\prime}\right)$, see [8] for further details.

In complete analogy to the open bosonic string, it is natural to organize (3.6) and (3.7) in a double-expansion w.r.t. $\alpha^{\prime}$ and transcendental weight. While the kinematic factors $B\left(\ldots ; \alpha^{\prime}\right)$ with an expansion as in $(2.8)$ only involve rational coefficients, the $\alpha^{\prime}$ corrections from $G\left(\alpha^{\prime}\right)$ still enjoy uniform transcendentality. At fixed order in $\alpha^{\prime}$, the leading-transcendentality part is again obtained by truncating $B\left(\ldots, \alpha^{\prime}\right) \rightarrow A_{\mathrm{YM}}(\ldots)$ and therefore identical in (3.5), (3.6) and (3.7). Hence, we have shown that, at leading transcendentality, gravitational tree-level interactions are universal to the bosonic, heterotic and type-II closed-string theories.

\subsection{Universality of UV completion}

It is instructive to consider the four-dimensional spinor helicity form of $B(1,2,3,4)$. For different helicity choices, it takes on the following simple form:

$$
\begin{aligned}
& B\left(1^{-}, 2^{-}, 3^{-}, 4^{-}\right)=-u \frac{\langle 14\rangle\langle 23\rangle}{[14][23]}\left(\frac{1}{(1+s)}+\frac{1}{(1+t)}+\frac{1}{(1+u)}-1\right) \\
& B\left(1^{-}, 2^{-}, 3^{-}, 4^{+}\right)=u \frac{\langle 12\rangle\langle 23\rangle[24]}{[12][23]\langle 24\rangle} \\
& B\left(1^{-}, 2^{-}, 3^{+}, 4^{+}\right)=u \frac{\langle 12\rangle^{2}}{\langle 34\rangle^{2}}\left(\frac{1}{(1+s)}-1\right) .
\end{aligned}
$$

For MHV amplitudes we will only need the last form. Thus the MHV amplitude in closed string theories can be written as

$$
\mathcal{M}_{4}^{S}\left(1^{-}, 2^{-}, 3^{+}, 4^{+}\right)=\langle 12\rangle^{4}[34]^{4} f(s, t, u)
$$

with the function $f(s, t, u)$ given as:

$$
\begin{aligned}
\text { Super } \quad f(s, t, u) & =\frac{\Gamma[1-s] \Gamma[1-u] \Gamma[1-t]}{\Gamma[1+s] \Gamma[1+u] \Gamma[1+t]}\left(\frac{-1}{s t u}\right) \\
\text { Heterotic } \quad f(s, t, u) & =\frac{\Gamma[1-s] \Gamma[1-u] \Gamma[1-t]}{\Gamma[1+s] \Gamma[1+u] \Gamma[1+t]}\left(\frac{-1}{s t u}+\frac{1}{s(1+s)}\right) \\
\text { Bosonic } \quad f(s, t, u) & =\frac{\Gamma[1-s] \Gamma[1-u] \Gamma[1-t]}{\Gamma[1+s] \Gamma[1+u] \Gamma[1+t]}\left(\frac{-1}{s t u}+\frac{2}{s(1+s)}-\frac{t u}{s(1+s)^{2}}\right) .
\end{aligned}
$$

Note that there is a universal $-\frac{1}{\text { stu }}$ piece, which in the low energy is simply yields the tree-level Einstein-Hilbert graviton amplitudes. For the heterotic string the extra term correspond to the presence of $R^{2} \phi$ operator, where $\phi$ is the dilaton. Note that the apparent Tachyon pole is cancelled by the zero at $s=-1$ in the gamma function produces. 
For the bosonic string, the two terms stem from $R^{2} \phi$ and $\left(R^{3}, R^{2} \tau\right)$ respectively, where $\tau$ is the Tachyon.

Thus we see that the UV completion of the the tree-level Einstein-Hilbert graviton amplitude is in fact unique in all perturbative string theories. The low energy amplitudes that stem from the presence of three-point higher-dimensional operators are completed separately. Note that the uniqueness of the UV completion for Einstein-Hilbert, follows directly from our conjectural form of the bosonic tree function in eq. (2.7), which has been proven up to seven-points. It will be extremely interesting to see whether the additional terms for heterotic and bosonic string graviton amplitudes at higher points can also be written as separate completion for tree-amplitudes of higher dimensional operators. We leave this for future study.

\section{Conclusions}

In this letter, tree-level amplitudes in all perturbative open- and closed-string theories are argued to have universal leading-transcendental parts in their $\alpha^{\prime}$-expansions. Manifest universality can be achieved by casting the world-sheet correlators of the bosonic open string into the same basis of disk integrals as the superstring, augmented with $\alpha^{\prime}$-dependent kinematic factors. We have explicitly shown that such a reorganization can be achieved up to seven points, and the conjectural all-multiplicity extension is relegated to future work [8]. Generalizations to closed-string interactions in bosonic, heterotic and type-II theories directly follow from the KLT-relations. These universality results have greatly facilitated the construction of matrix elements for counterterms in half-maximal supergravity [30].

It would be interesting to apply the same organizing principles to massive-state scattering. We expect the same basis of disk integrals to capture tree amplitudes among any combination of massive open-string resonances. Moreover, the structure of (3.5) is believed to apply to closed-string trees among massive resonances upon appropriate replacements of $A_{\mathrm{YM}}$ and $\tilde{A}_{\mathrm{YM}}$.

Finally the conjectural form of eq. (2.7) leads to the interesting conclusion that the UV completion of pure Einstein-Hilbert gravity is in fact unique amongst perturbative string theory. The difference lies in the presence of separate terms in the amplitude that correspond to UV completions of higher dimensional operators of the form $R^{2}, R^{2} \phi$ and $R^{3}$. This structure is straight forward at four-points, and it will be interesting to see if the latter persists to higher points.

\section{Acknowledgments}

We are grateful to Johannes Brödel, Paolo Di Vecchia, Michael Green and Henrik Johansson for inspiring discussions and valuable comments on a draft of the manuscript. Also, Massimo Bianchi and Andrea Guerrieri are thanked for enlightening discussions. Y-t would like to thank Nima Arkani-Hamed for the interesting observation of uniqueness in UV completion of Einstein-Hilbert gravity. Y-t.H. is supported by MOST under the grant No. 103-2112-M-002-025-MY3, and O.S. is grateful to the Università di Roma Tor Vergata for kind hospitality during finalization of this work. 
Open Access. This article is distributed under the terms of the Creative Commons Attribution License (CC-BY 4.0), which permits any use, distribution and reproduction in any medium, provided the original author(s) and source are credited.

\section{References}

[1] D.J. Gross and P.F. Mende, The High-Energy Behavior of String Scattering Amplitudes, Phys. Lett. B 197 (1987) 129 [inSPIRE].

[2] D. Amati, M. Ciafaloni and G. Veneziano, Classical and Quantum Gravity Effects from Planckian Energy Superstring Collisions, Int. J. Mod. Phys. A 3 (1988) 1615 [INSPIRE].

[3] X.O. Camanho, J.D. Edelstein, J. Maldacena and A. Zhiboedov, Causality Constraints on Corrections to the Graviton Three-Point Coupling, JHEP 02 (2016) 020 [arXiv:1407.5597] [INSPIRE].

[4] N. Arkani-Hamed, Y-t. Huang and Z-C. Huang, to appear.

[5] E. Witten, String theory dynamics in various dimensions, Nucl. Phys. B 443 (1995) 85 [hep-th/9503124] [INSPIRE].

[6] C.M. Hull and P.K. Townsend, Unity of superstring dualities, Nucl. Phys. B 438 (1995) 109 [hep-th/9410167] [INSPIRE].

[7] H. Kawai, D.C. Lewellen and S.H.H. Tye, A Relation Between Tree Amplitudes of Closed and Open Strings, Nucl. Phys. B 269 (1986) 1 [INSPIRE].

[8] Y-t. Huang, O. Schlotterer and C. Wen, to appear.

[9] C.R. Mafra, O. Schlotterer and S. Stieberger, Complete N-Point Superstring Disk Amplitude I. Pure Spinor Computation, Nucl. Phys. B 873 (2013) 419 [arXiv:1106.2645] [InSPIRE].

[10] J. Broedel, O. Schlotterer, S. Stieberger and T. Terasoma, All order $\alpha^{\prime}$-expansion of superstring trees from the Drinfeld associator, Phys. Rev. D 89 (2014) 066014 [arXiv: 1304.7304] [INSPIRE].

[11] N.E.J. Bjerrum-Bohr, P.H. Damgaard and P. Vanhove, Minimal Basis for Gauge Theory Amplitudes, Phys. Rev. Lett. 103 (2009) 161602 [arXiv:0907.1425] [InSPIRE].

[12] S. Stieberger, Open $\&$ Closed vs. Pure Open String Disk Amplitudes, arXiv:0907.2211 [INSPIRE].

[13] D. Oprisa and S. Stieberger, Six gluon open superstring disk amplitude, multiple hypergeometric series and Euler-Zagier sums, hep-th/0509042 [INSPIRE].

[14] S. Stieberger and T.R. Taylor, Multi-Gluon Scattering in Open Superstring Theory, Phys. Rev. D 74 (2006) 126007 [hep-th/0609175] [INSPIRE].

[15] R. Kleiss and H. Kuijf, Multi-Gluon Cross-sections and Five Jet Production at Hadron Colliders, Nucl. Phys. B 312 (1989) 616 [inSPIRE].

[16] V. Del Duca, L.J. Dixon and F. Maltoni, New color decompositions for gauge amplitudes at tree and loop level, Nucl. Phys. B 571 (2000) 51 [hep-ph/9910563] [inSPIRE].

[17] Z. Bern, J.J.M. Carrasco and H. Johansson, New Relations for Gauge-Theory Amplitudes, Phys. Rev. D 78 (2008) 085011 [arXiv:0805.3993] [INSPIRE].

[18] J. Broedel, O. Schlotterer and S. Stieberger, Polylogarithms, Multiple Zeta Values and Superstring Amplitudes, Fortsch. Phys. 61 (2013) 812 [arXiv:1304.7267] [INSPIRE]. 
[19] C.R. Mafra, O. Schlotterer, S. Stieberger and D. Tsimpis, Six Open String Disk Amplitude in Pure Spinor Superspace, Nucl. Phys. B 846 (2011) 359 [arXiv: 1011.0994] [InSPIRE].

[20] J. Broedel and L.J. Dixon, Color-kinematics duality and double-copy construction for amplitudes from higher-dimension operators, JHEP 10 (2012) 091 [arXiv:1208.0876] [INSPIRE].

[21] S. Stieberger and T.R. Taylor, Closed String Amplitudes as Single-Valued Open String Amplitudes, Nucl. Phys. B 881 (2014) 269 [arXiv:1401.1218] [INSPIRE].

[22] O. Schlotterer and S. Stieberger, Motivic Multiple Zeta Values and Superstring Amplitudes, J. Phys. A 46 (2013) 475401 [arXiv:1205.1516] [INSPIRE].

[23] J. Broedel, O. Schlotterer and S. Stieberger, $\alpha^{\prime}$-expansion of open superstring amplitudes, http://mzv.mpp.mpg.de.

[24] S. Stieberger, Constraints on Tree-Level Higher Order Gravitational Couplings in Superstring Theory, Phys. Rev. Lett. 106 (2011) 111601 [arXiv:0910.0180] [INSPIRE].

[25] S. Stieberger, Closed superstring amplitudes, single-valued multiple zeta values and the Deligne associator, J. Phys. A 47 (2014) 155401 [arXiv:1310.3259] [InSPIRE].

[26] O. Schnetz, Graphical functions and single-valued multiple polylogarithms, Commun. Num. Theor. Phys. 08 (2014) 589 [arXiv:1302.6445] [INSPIRE].

[27] F. Brown, Single-valued Motivic Periods and Multiple Zeta Values, SIGMA 2 (2014) e25 [arXiv: 1309.5309] [INSPIRE].

[28] N.E.J. Bjerrum-Bohr, P.H. Damgaard, T. Sondergaard and P. Vanhove, The Momentum Kernel of Gauge and Gravity Theories, JHEP 01 (2011) 001 [arXiv:1010.3933] [INSPIRE].

[29] Z. Bern, L.J. Dixon, M. Perelstein and J.S. Rozowsky, Multileg one loop gravity amplitudes from gauge theory, Nucl. Phys. B 546 (1999) 423 [hep-th/9811140] [INSPIRE].

[30] Y.-t. Huang and C. Wen, Soft theorems from anomalous symmetries, JHEP 12 (2015) 143 [arXiv: 1509.07840] [INSPIRE]. 